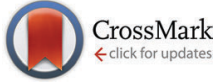

Cite this: Phys. Chem. Chem. Phys., 2016, 18, 5964

Received 29th October 2015, Accepted 27th January 2016

DOI: $10.1039 / \mathrm{c} 5 \mathrm{cp} 06598 \mathrm{~d}$

www.rsc.org/pccp

\section{Electric dipole moments and chemical bonding of diatomic alkali-alkaline earth molecules $\dagger$}

\author{
Johann V. Pototschnig,* Andreas W. Hauser and Wolfgang E. Ernst
}

\begin{abstract}
We investigate the properties of alkali-alkaline earth diatomic molecules in the lowest $\Sigma^{+}$states of the doublet and quartet multiplicity by ab initio calculations. In all sixteen cases studied, the permanent electric dipole moment points in opposite directions for the two spin states. This peculiarity can be explained by molecular orbital theory. We further discuss dissociation energies and bond distances. We analyze trends and provide an empirically motivated model for the prediction of the permanent electric dipole moment for combinations of alkali and alkaline earth atoms not studied in this work.
\end{abstract}

\section{Introduction}

In the last years ultracold molecules have attracted a lot of attention due to their outstanding properties. ${ }^{1-6}$ The low temperature reduces the decoherence, and ensembles of ultracold molecules can be used to simulate quantum systems ${ }^{7-12}$ or perform quantum computations. ${ }^{13}$ Most degrees of freedom are frozen out at these temperatures, which allows precise control of chemical reactions. ${ }^{14-16}$ Another advantage is the reduction of the linewidth and noise in measurements of fundamental physical properties and constants ${ }^{17}$ such as the proton to electron mass ratio, ${ }^{18-20}$ the electron electric dipole moment ${ }^{21,22}$ or the fine structure constant. ${ }^{23,24}$

The first molecules produced in the ultracold temperature regime were homonuclear alkali-dimers, ${ }^{25-28}$ which do not have a permanent electric dipole moment. A few years later, ultracold heteronuclear alkali-dimers were produced. ${ }^{29-34}$ Their permanent electric dipole moment gives experimentalists an additional handle for manipulation via electric fields. In this sense, the next logical step is the production of ultracold molecules with a dipole moment plus an additional magnetic moment, originating from an unpaired electron spin. This magnetic moment causes additional interactions and enables control of these molecules via magnetic fields. Currently, the most promising candidates are alkali (AK)-alkaline earth (AKE) and $\mathrm{AK}-\mathrm{Yb}$ diatomic molecules because the involved atoms are well under control in ultracold atomic physics. Bose-Einstein condensation, for example, has been achieved for most AK atoms, ${ }^{35}$

Graz University of Technology, Institute of Experimental Physics, Petersgasse 16, 8010 Graz, Austria. E-mail: johann.pototschnig@tugraz.at,

wolfgang.ernst@tugraz.at; Tel: +43 316 873-8152

$\dagger$ Electronic supplementary information (ESI) available: Several tables regarding the calculations and their results as well as orbital pictures. See DOI: 10.1039/c5cp06598d
$\mathrm{Ca},{ }^{36} \mathrm{Sr}^{37}$ and $\mathrm{Yb}^{38}$ In a series of recent studies combining experimental and computational efforts ${ }^{39-43}$ we found permanent electric dipole moments (PEDM) with opposite signs in the lowest $\Sigma^{+}$states of the doublet and the quartet multiplicity for AK-AKE molecules. In this work we analyze this behavior and discuss the trends in greater detail. The magnitude of the permanent electric dipole moment (PEDM) is of special importance for future applications. A significant value is necessary to align molecules in an optical lattice, ${ }^{44,45}$ to control interactions with an external microwave field, ${ }^{4,11,46}$ and it might even be useful to reach low vibrational levels. ${ }^{47}$

Early on, there has been interest in the prediction of dipole moments for alkali halides and alkaline earth halides. These two groups of molecules with predominantly ionic bonding could successfully be described by electrostatic interaction models. ${ }^{48-50}$ The Rittner model ${ }^{48}$ for alkali halides assumes a complete charge transfer of one electron and considers the polarizabilities of the ions. Törring et al. ${ }^{51,52}$ applied a similar model to AKE halides. More sophisticated computations using ligand field theory were performed for AKE halides ${ }^{53}$ and lanthanum containing diatomics. ${ }^{54,55} \mathrm{~A}$ model taking orbital mixing into account was applied to $\mathrm{SrBr}$ in ref. 56. However, these techniques assume a transfer of at least one electron and can therefore not be applied to AK-AKE molecules. Recently, an empirical model for the relation between dipole moment and vibrational frequencies was presented. ${ }^{57}$

There are a few theoretical and experimental studies concerned with the properties of AK-AKE molecules. Bauschlicher et al. ${ }^{58}$ performed one of the first calculations. Two theoretical studies examined the ground state of Li-AKE molecules by coupled cluster calculations. ${ }^{59,60}$ The ground states of neutral $\mathrm{AK}-\mathrm{Sr}$ molecules and several states of the molecular ions have been investigated by a configuration interaction method. ${ }^{61,62}$ In ref. 63, the ground states of $\mathrm{AK}-\mathrm{Mg}$ diatomic molecules were 
calculated at the coupled cluster level of theory. Using the same approach, Gopakumar et al. ${ }^{45}$ recently investigated the AK-AKE molecules $(\mathrm{Na}, \mathrm{K}, \mathrm{Rb})-(\mathrm{Ca}, \mathrm{Sr})$. The combination of barium with an alkali atom has also been considered in the ground state. ${ }^{64}$ Some older studies analyzed the ground and several excited states of $\mathrm{LiBa}^{65,66}$ and $\mathrm{NaBa}{ }^{67}$ The most thoroughly studied representative of these diatomic molecules is $\mathrm{LiCa}$, with experimental, ${ }^{68-70}$ theoretical $^{71,72}$ and combined investigations. ${ }^{39,73}$ The first realization of a combined quantum degenerate mixture of $\mathrm{Rb}$ and $\mathrm{Sr}^{74}$ was followed by a series theoretical and experimental publications. ${ }^{40-42,75-77}$ The excited states of $\mathrm{LiSr}^{72} \mathrm{RbBa}^{78}$ LiMg, ${ }^{79}$ and $\mathrm{RbCa}^{43}$ were calculated recently.

In this study, we investigate the energetically lowest electronic states of neutral $\mathrm{AK}(\mathrm{Li}, \mathrm{Na}, \mathrm{K}, \mathrm{Rb})-\mathrm{AKE}(\mathrm{Be}, \mathrm{Mg}, \mathrm{Ca}, \mathrm{Sr}$ ) molecules in the doublet and quartet manifold. Throughout the article, the coordinate system is defined such that the AKE atom is on the negative side of the axis and the AK atom on the positive side. Therefore, a positive value of the PEDM corresponds to a dipole moment pointing in axis direction from the AKE to the AK atom (i.e., the center of the negative charge lies closer to the AKE atom).

This manuscript is structured as follows: in the next section details of the computational method are given, then an overview of the results and a comparison to previous results. After discussing trends for AK-AKE molecules we give a qualitative description of the situation for the lowest $\Sigma^{+}$states in AK-AKE molecules. Finally, we present an empirical model in Section 3.4.

\section{Computational details}

All $a b$ initio calculations were carried out with the MOLPRO ${ }^{80}$ software package. A multiconfigurational self consistent field calculation (MCSCF ${ }^{81,82}$ ) was performed, followed by multireference configuration interaction including Davidson correction ( $\mathrm{MRCI}^{83,84}$ ). The quintuple-zeta basis set family of Peterson et al. covers most atoms of our study and was used where possible. All electrons basis sets ${ }^{85}$ were applied for $\mathrm{Li}, \mathrm{Na}$, Be and $\mathrm{Mg}$. For the heavier atoms the corresponding effective core potential basis sets $^{86}$ of Peterson were combined with the recommended effective core potentials $^{87,88}$ of Lim et al. For $\mathrm{K}$ and $\mathrm{Rb}$, however, no Peterson basis sets were available. In these cases, we fell back on the basis sets provided by Lim, ${ }^{87,88}$ decontracted them, and added several diffuse functions to obtain a description of similar accuracy. $\neq$ The same strategy has recently been applied to $\mathrm{RbCa},{ }^{43}$ yielding excitation energies in good agreement with experimental measurements.

We further applied core polarization potentials (CPPs) as introduced by Müller and Meyer ${ }^{89}$ due to their importance for correct magnitudes of the permanent electric dipole moments ${ }^{90,91}$ (see Fig. 1). The parameters of the core polarization potential are given (see Table 1) and were optimized for our effective core potentials and basis sets. This approach only allows basis

\# The basis sets were completely decontracted and additional basis functions were added: K[s: 0.0037, 0.0017; p: 0.0016, 0.00062; d: 4.34, 0.011; f: 0.0296]; Rb[s: 0.0036, p: 0.0042 , d: $0.0116,2.860$, f: 0.0624 g: 0.33].

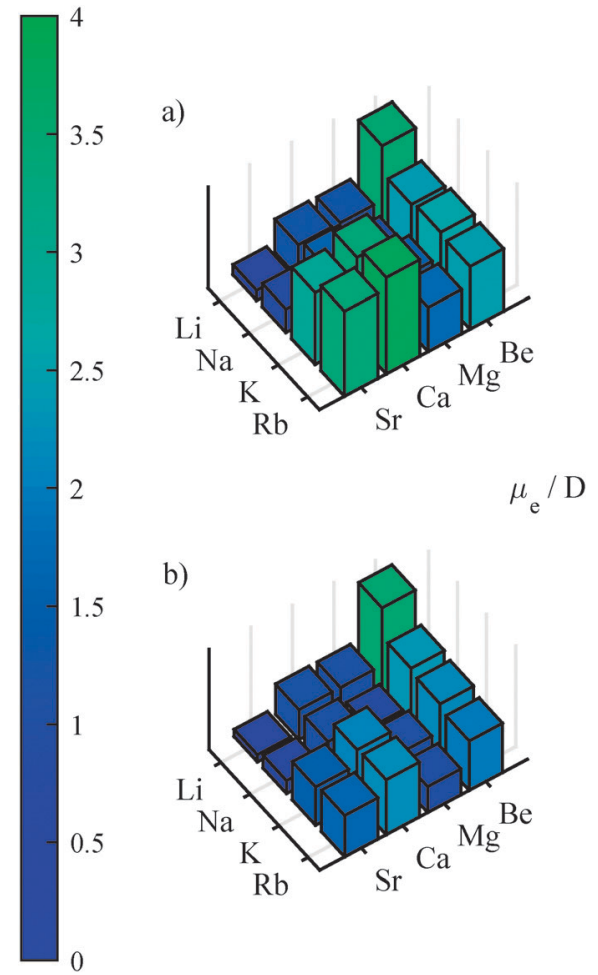

Fig. 1 The permanent electric dipole moment of the doublet ground state at its equilibrium distance, obtained at the MCSCF + MRCI level of theory, without (a) and with (b) the inclusion of core polarization potentials.

Table 1 Parameters of the CPPs used in this study; $\alpha_{\text {core }}$ is the dipole polarizability and $\rho$ is the cutoff parameter. The CPPs account for the polarizability of the innermost shells. These parameters have been adjusted for the best reproduction of atomic excitation energies

\begin{tabular}{llllll}
\hline $\mathrm{AK}$ & $\alpha_{\text {core }} /$ a.u. & $\rho / \AA^{-2}$ & AKE & $\alpha_{\text {core }} /$ a.u. & $\rho / \AA^{-2}$ \\
\hline $\mathrm{Na}$ & 1.00 & 1.27 & $\mathrm{Mg}$ & 1.20 & 0.60 \\
$\mathrm{~K}$ & 5.61 & 0.46 & $\mathrm{Ca}$ & 7.16 & 0.25 \\
$\mathrm{Rb}$ & 9.74 & 0.35 & $\mathrm{Sr}$ & 9.28 & 0.28
\end{tabular}

functions with an angular quantum number below 5 . Therefore, basis functions with higher quantum numbers were neglected. No core polarization was added for $\mathrm{Li}$ and Be due to their small cores and large energy difference between the core orbitals and valence orbitals compared to the other elements. With this approach the energy difference between the first excited state $\left({ }^{2} \mathrm{P}_{\mathrm{AK}} /{ }^{3} \mathrm{P}_{\mathrm{AKE}}\right)$ and the ground state agrees within $150 \mathrm{~cm}^{-1}(1 \%)$ with the atomic values ${ }^{92}$ in the asymptotic limit.

It is known that the correlation or polarization of the core electrons has a significant influence on the dipole moment. ${ }^{90,91}$ The importance of this contribution can be estimated by looking at Fig. 1, where the results without (a) and with (b) core polarization potentials are compared. Typically, the PEDM is overestimated if the polarization of the core is neglected, which agrees well with the observations of ref. 90. This error increases with atomic mass. The largest deviation was found for RbSr, where the dipole moment is reduced by $1.65 \mathrm{D}$ to about half its value after inclusion of core polarization. The PEDMs for the 
computations including core polarization potentials (see plot $\mathrm{b}$ of Fig. 1) were obtained by finite field calculations, $\S$ which we carried out for different field strengths varying from $10^{-5}$ to $5 \times 10^{-3}$ a.u. for all 16 molecules. For each field strength the PEDMs and polarizabilities were computed by three point and five point finite field approximations and compared to each other to estimate the size of numerical error. The values were then taken where the smallest deviation was observed. (The values and the field strengths for their calculation are listed in the ESI.†)

The PEDMs for the lowest $\Sigma^{+}$state of the quartet multiplicity were also changed significantly by the core polarization potential, especially for heavy molecules, but without a clear trend. An improvement of the potential depth of LiCa was achieved by a new set of recently adjusted core polarization parameters. The potential depth for LiCa obtained in this work $\left(2613 \mathrm{~cm}^{-1}\right)$ now agrees better with the experimental value $\left(2605 \mathrm{~cm}^{-1}\right)^{69}$ than our previous calculation. ${ }^{39}$ However, part of the improvement might originate also from the slightly different basis set. On average, the equilibrium distance was reduced by the core polarization potential, although some minor deviations occurred, especially for the quartet states.

In the MCSCF and MRCI calculation the same active space was applied, but it was necessary to adapt it to the different basis sets and effective core potentials. Details on the size and definition of the active space for the various diatomics can be found in the ESI. $\uparrow$ Note that the lowest $\Sigma^{+}$state in the quartet multiplicity is an electronically excited state accompanied by several excited states of the doublet manifold. Therefore, several excited states were calculated in both spin configurations to obtain a meaningful description of this state. In a combined MCSCF calculation 16 to 32 states of doublet multiplicity and 3 or 8 states of quartet multiplicity were optimized. The number of states was kept the same in the MRCI calculation for the quartet multiplicity, but the number of doublet states was reduced to 12-17 states. With this setup for the state-averaged calculations we obtained smooth potential energy curves up to an internuclear separation of $10 \AA$. At this distance, the deviation for the ground state from the asymptotic value is only $10 \mathrm{~cm}^{-1}$ for light atoms and about $25 \mathrm{~cm}^{-1}$ for heavy atoms (ESI $\dagger$ ).

\section{Results}

\subsection{Overview}

The properties of $16 \mathrm{AK}-\mathrm{AKE}$ molecules have been calculated by MCSCF + MRCI and the values for the ground state are listed in Table 2. Results for the lowest $\Sigma^{+}$state in the quartet multiplicity are given in Table 3. Besides the ab initio calculations performed in this work, several other theoretical predictions as well as three experimental studies are listed in Table 2. Overall agreement between the various studies is good except for significant differences in dissociation energies. LiCa, the best studied

$\S$ This was necessary because the dipole moments determined by the MRCI routine in MOLPRO do not include the dipole moment of the polarized core. However, the difference between the dipole moments determined by the routine and the finite field calculations are smaller than $0.05 \mathrm{D}$ in all cases.
Table 2 Results for the ${ }^{2} \Sigma^{+}$ground state, obtained at the MRCl level of theory, including core polarization potentials. Where available, values from the literature are given for comparison

\begin{tabular}{|c|c|c|c|c|c|c|}
\hline Mol. & Ref. & $\mu_{\mathrm{e}} / \mathrm{D}$ & $r_{\mathrm{e}} / \AA ̊ \AA$ & $D_{\mathrm{e}} / \mathrm{cm}^{-1}$ & $\omega_{\mathrm{e}} / \mathrm{cm}^{-1}$ & $\alpha_{\|}{ }^{a} /$ a.u. \\
\hline \multirow[t]{4}{*}{ LiBe } & This work & 3.47 & 2.60 & 2427 & 313 & 365 \\
\hline & 60 & 3.58 & 2.58 & 2406 & 300 & \\
\hline & 58 & & 2.61 & 2339 & 300 & \\
\hline & $93^{b}$ & & 2.59 & & 295 & \\
\hline \multirow[t]{7}{*}{$\mathrm{LiMg}$} & This work & 1.18 & 3.10 & 1538 & 181 & 470 \\
\hline & 79 & 1.22 & 3.10 & 1395 & 174 & \\
\hline & 63 & 1.02 & 3.10 & 1445 & 180 & \\
\hline & 59 & $0.86^{c}$ & 3.12 & 1332 & 187 & 482 \\
\hline & 60 & 1.12 & 3.11 & 1432 & 206 & \\
\hline & 58 & & 3.11 & 1613 & 183 & \\
\hline & $94^{b}$ & & & 1331 & 190 & \\
\hline \multirow[t]{8}{*}{ LiCa } & This work & 1.19 & 3.39 & 2613 & 200 & 588 \\
\hline & 39 & & 3.34 & 2883 & 203 & \\
\hline & 72 & & 3.40 & 2131 & 206 & \\
\hline & 59 & $1.10^{c}$ & 3.40 & 2260 & 210 & 599 \\
\hline & 60 & 1.12 & 3.36 & 2607 & 207 & \\
\hline & 73 & & 3.40 & 2178 & 204 & \\
\hline & 71 & & 3.30 & 2355 & 196 & \\
\hline & $69^{b}$ & & 3.36 & 2605 & 202 & \\
\hline \multirow[t]{5}{*}{ LiSr } & This work & 0.28 & 3.57 & 2471 & 181 & 653 \\
\hline & 72 & & 3.58 & 2074 & 181 & \\
\hline & 59 & $0.31^{c}$ & 3.53 & 2223 & 196 & 640 \\
\hline & 60 & 0.24 & 3.55 & 2401 & 184 & \\
\hline & 61 & 0.34 & 3.48 & 2587 & 185 & \\
\hline \multirow[t]{2}{*}{$\mathrm{NaBe}$} & This work & 2.33 & 2.97 & 1291 & 173 & 397 \\
\hline & 58 & & 3.05 & 1129 & 164 & \\
\hline \multirow[t]{3}{*}{$\mathrm{NaMg}$} & This work & 0.86 & 3.47 & 946 & 90 & 432 \\
\hline & 63 & 0.72 & 3.53 & 825 & 83 & \\
\hline & 58 & & 3.56 & 887 & 85 & \\
\hline \multirow[t]{2}{*}{$\mathrm{NaCa}$} & This work & 1.18 & 3.67 & 1792 & 103 & 577 \\
\hline & 45 & 1.01 & 3.72 & 1453 & 97 & 594 \\
\hline \multirow[t]{3}{*}{$\mathrm{NaSr}$} & This work & 0.51 & 3.84 & 1728 & 87 & 636 \\
\hline & 45 & 0.49 & 3.89 & 1441 & 82 & 633 \\
\hline & 61 & 0.62 & 3.82 & 1597 & 85 & \\
\hline $\mathrm{KBe}$ & This work & 2.22 & 3.51 & 920 & 122 & 628 \\
\hline \multirow[t]{2}{*}{$\mathrm{KMg}$} & This work & 1.08 & 3.99 & 779 & 64 & 656 \\
\hline & 63 & 0.83 & 4.09 & 647 & 58 & \\
\hline \multirow[t]{2}{*}{$\mathrm{KCa}$} & This work & 2.12 & 4.20 & 1455 & 71 & 869 \\
\hline & 45 & 1.67 & 4.32 & 974 & 61 & 892 \\
\hline \multirow[t]{3}{*}{$\mathrm{KSr}$} & This work & 1.52 & 4.39 & 1358 & 57 & 925 \\
\hline & 45 & 1.27 & 4.53 & 964 & 48 & 942 \\
\hline & 61 & 1.52 & 4.41 & 1166 & 52 & \\
\hline $\mathrm{RbBe}$ & This work & 1.97 & 3.70 & 816 & 104 & 631 \\
\hline \multirow[t]{2}{*}{$\mathrm{RbMg}$} & This work & 1.04 & 4.17 & 744 & 54 & 664 \\
\hline & 63 & 0.79 & 4.29 & 586 & 47 & \\
\hline \multirow[t]{3}{*}{$\mathrm{RbCa}$} & This work & 2.19 & 4.37 & 1337 & 57 & 922 \\
\hline & 43 & 2.54 & 4.37 & 1406 & 58 & \\
\hline & 45 & 1.75 & 4.53 & 921 & 49 & 961 \\
\hline \multirow[t]{6}{*}{$\mathrm{RbSr}$} & This work & 1.64 & 4.56 & 1279 & 43 & 972 \\
\hline & 42 & 1.80 & 4.59 & 1274 & 42 & \\
\hline & 77 & & 4.67 & 1041 & 38 & \\
\hline & 76 & & 4.67 & 1018 & 36 & \\
\hline & 45 & 1.41 & 4.72 & 916 & 36 & 1009 \\
\hline & 61 & 1.54 & 4.60 & 1073 & 32 & \\
\hline
\end{tabular}

${ }^{a}$ Polarizabilities along the internuclear axis for the diatomic molecules. ${ }^{b}$ Experimental results. ${ }^{c} d_{0}$ values are given. 
Table 3 Results for the lowest ${ }^{4} \Sigma^{+}$state, obtained at the MRCl level of theory, including core polarization potentials. Where available, values from the literature are given for comparison

\begin{tabular}{llllllr}
\hline Mol. & Ref. & $\mu_{\mathrm{e}} / \mathrm{D}$ & $r_{\mathrm{e}} / \AA$ & $D_{\mathrm{e}} / \mathrm{cm}^{-1}$ & $\omega_{\mathrm{e}} / \mathrm{cm}^{-1}$ & $\alpha_{\|}{ }^{a} / \mathrm{a} . \mathrm{u}$. \\
\hline $\mathrm{LiBe}$ & This work & -0.61 & 5.51 & 59 & 25 & 223 \\
$\mathrm{LiMg}$ & This work & -2.52 & 4.72 & 222 & 32 & 423 \\
$\mathrm{LiCa}$ & This work & -4.58 & 4.59 & 522 & 55 & 1031 \\
& 39 & & 4.33 & 608 & 67 & \\
& 72 & & 5.01 & 265 & 30 & \\
$\mathrm{LiSr}$ & This work & -6.61 & 4.59 & 692 & 61 & 1340 \\
& 72 & & 4.60 & 401 & 47 & \\
$\mathrm{NaBe}$ & This work & -0.45 & 5.84 & 50 & 18 & 229 \\
$\mathrm{NaMg}$ & This work & -0.99 & 5.71 & 131 & 21 & 326 \\
$\mathrm{NaCa}$ & This work & -1.62 & 5.74 & 237 & 24 & 686 \\
$\mathrm{NaSr}$ & This work & -2.00 & 5.81 & 287 & 23 & 1200 \\
$\mathrm{KBe}$ & This work & -0.72 & 5.99 & 48 & 16 & 300 \\
$\mathrm{KMg}$ & This work & -1.58 & 5.73 & 157 & 20 & 338 \\
$\mathrm{KCa}$ & This work & -2.50 & 5.73 & 343 & 26 & 950 \\
$\mathrm{KSr}$ & This work & -2.99 & 5.95 & 376 & 22 & 1523 \\
$\mathrm{RbBe}$ & This work & -0.79 & 6.03 & 47 & 15 & 363 \\
$\mathrm{RbMg}$ & This work & -1.66 & 5.84 & 170 & 18 & 645 \\
$\mathrm{RbCa}$ & This work & -2.13 & 6.05 & 316 & 19 & 922 \\
& 43 & & 5.98 & 336 & 19 & \\
$\mathrm{RbSr}$ & This work & -2.66 & 6.15 & 343 & 16 & 1491 \\
& 42 & & 6.17 & 390 & 16 & \\
& 77 & & 6.25 & 329 & 15 & \\
& 77 & & 6.15 & 336 & 15 &
\end{tabular}

${ }^{a}$ Polarizabilities along the internuclear axis for the diatomic molecules.

molecule in the literature, with data available from 8 different sources, shows a large variation of the dissociation energy. Our value and the value of ref. 60 agree best with recent experimental findings. ${ }^{69}$ Two older experimental results ${ }^{93,94}$ are also listed in Table 2. A recent experiment of our group ${ }^{40}$ yielded a vibrational constant $\left(\omega_{\mathrm{e}}\right)$ of $(42 \pm 5) \mathrm{cm}^{-1}$ for $\mathrm{RbSr}$, which is in good agreement with the theoretical results in Table 2 . The calculated PEDMs are all positive and differ on average by $0.16 \mathrm{D}(13 \%)$ from previous computations, with the largest difference observed for the PEDM of KCa in ref. 45. Note that the PEDM values listed in Table 2 were evaluated for the respective equilibrium distance. When zero-point motion is considered, the PEDM is on average reduced by $0.03 \mathrm{D}$ for the doublet states and by $0.06 \mathrm{D}$ for the quartet states (details are provided in the ESI $\dagger$ ). The difference to other equilibrium distances is on average $0.05 \AA$ (1\%) with the largest deviations occurring for $\mathrm{RbSr}$ and $\mathrm{RbCa}$ when compared to ref. 45. This deviation in the latter cases also explains the slight difference in the PEDM as the listed values are taken at the equilibrium separation. A small disagreement can also be found between the parallel polarizabilities (along the internuclear axis) listed in Table 2 . Note that only the polarizabilities $\left(\alpha_{\|}\right)$at the equilibrium distances along the internuclear axis are given here. A more detailed treatment of polarizabilities and their experimental significance is provided in ref. 45 for AK-AKE molecules or in ref. 44 for AK-AK molecules.

A similar compilation of data for the lowest ${ }^{4} \Sigma^{+}$state is given in Table 3, although fewer theoretical and experimental values are available. $\uparrow$ In general, the equilibrium radius is larger and

I Note that this is not the lowest state in the quartet multiplicity. The $1^{4} \Pi$ state has the same asymptote but is more strongly bound than the $1^{4} \Sigma^{+}$state. the potential depth and vibrational constant are smaller than corresponding quantities for the ground state. For the polarizability no clear trend can be observed.

\subsection{Trends for AK-AKE molecules}

A diatomic molecule formed by a single alkali atom and a single alkaline earth atom comprises three valence electrons. If excitations of core electrons and ionizations are neglected, these three electrons can only form a doublet or a quartet state. The AK atom, providing one unpaired electron, is always in a doublet state in the asymptotic limit of separated atoms. In contrast, the AKE atom can be either in a singlet or in a triplet state. A doublet state of the molecule is therefore either realized by the combination of a singlet AKE atom with the doublet AK atom, or by the combination of a triplet AKE atom with an anti-aligned electron from the AK atom. Quartet multiplicity can only arise if a triplet state of the AKE atom is combined with an aligned electron on the AK atom. The ground state of the molecule must be a doublet state, since the lowest state of the AKE atom is of singlet character. Only an excited triplet state of the AKE can result in a quartet state. For the AKE atoms considered in this study (Be-Sr), this is a ${ }^{3} \mathrm{P}$ state with excitation energies between $22000 \mathrm{~cm}^{-1}$ and $14500 \mathrm{~cm}^{-1}$. For $\mathrm{Ba}$ and $\mathrm{Ra}$ the lowest excitation has ${ }^{3} \mathrm{D}$ character. The lowest excitation of the $\mathrm{AK}$ atom is a ${ }^{2} \mathrm{P}$ state with energies ranging from $12500 \mathrm{~cm}^{-1}$ to $17000 \mathrm{~cm}^{-1}$. The energy difference between the lowest excitation of the AKE $\left({ }^{3} \mathrm{P}\right)$ and the $\mathrm{AK}$ atom $\left({ }^{2} \mathrm{P}\right)$ is plotted in graphics (a) of Fig. 2. Note that there are

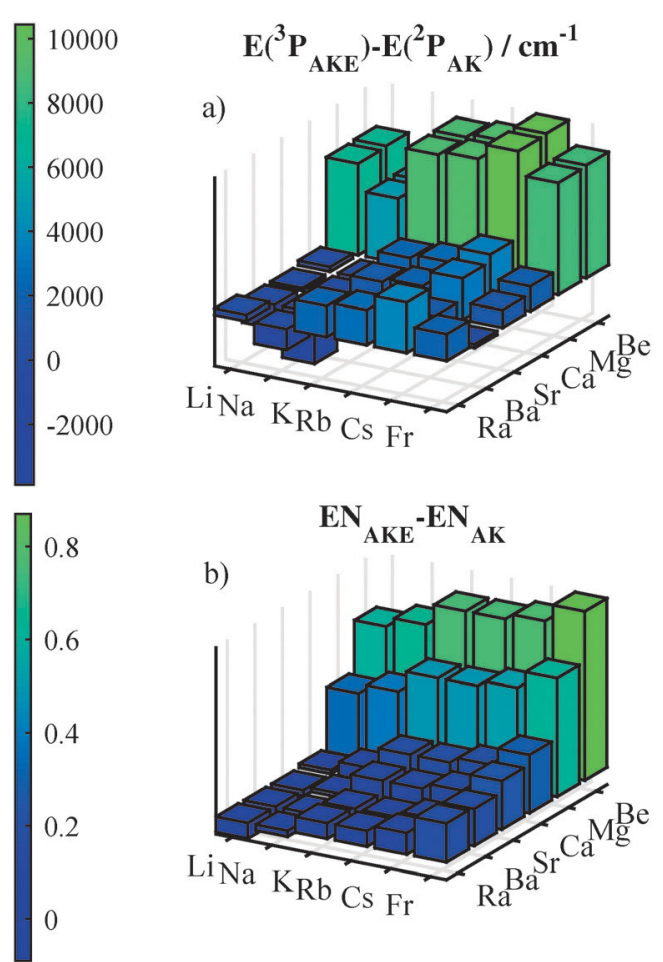

Fig. 2 Differences between AK and AKE atoms for two atomic properties are shown in the figure, in the upper plot energy differences of the first $\mathrm{P}$-excitations ${ }^{92}$ and in the lower plot the differences in the electronegativities according to Pauling's scale. ${ }^{96}$ 
negative values for combinations of light alkali atoms and heavy alkaline earth atoms. Rather than the ${ }^{3} \mathrm{D}$-excitations, which are the lowest ones, the ${ }^{3} \mathrm{P}$-excitations were used in Fig. 2 for $\mathrm{Ba}$ and Ra. In plot (b) of Fig. 2 the difference in electronegativities (ENs) is shown with a behavior similar to the differences in excitation energies. A positive value of the electronegativity difference indicates that the electron density is pulled towards the AKE atom. Only combinations of light AK atoms with heavy AKE atoms have negative differences. Within our test set only LiSr has a negative EN difference. The difference in excitation energy or electronegativities characterizes the direction and magnitude of the pull at the electron density. Additionally, the polarizability is important as it measures the flexibility of the electron density. The atomic values for the polarizability range from 37 to 248 a.u. for AKE atoms and from 164 to 313 a.u. for AK atoms, ${ }^{95}$ with an increasing value for heavier atoms. The change in the orbital of the AK atom can be expected to be larger than the change for the AKE atom if atoms of similar mass are combined. Such a behavior was observed and is discussed in Section 3.3.

Fig. 3 shows the PEDMs (a) and dissociation energies (b) for the ground state of the $16 \mathrm{AK}-\mathrm{AKE}$ molecules within our test set. The smallest PEDM was obtained for LiSr, but unlike the difference in ENs, it still is positive. The largest EN difference for the calculated molecules is found for the RbBe molecule, but LiBe shows the largest dipole moment. The increase by going from light to heavy $\mathrm{AK}$ atoms and $\mathrm{AKE}$ atoms as seen in the difference of ENs in plot (b) of Fig. 2 is not seen for the PEDMs. For KMg and RbMg small PEDMs are observable in Fig. 3. The small PEDMs for $\mathrm{Mg}$ containing molecules are probably related to the significantly smaller electronegativity difference for $\mathrm{Mg}$ than for Be, although they have similar differences in excitation
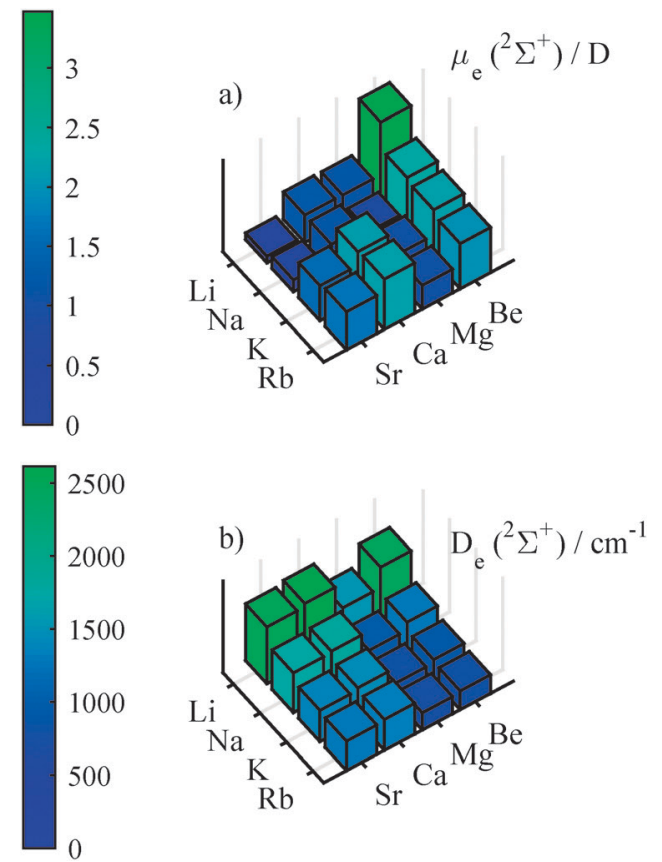

Fig. 3 The permanent electric dipole moment (a) and the potential depth (b) for several AK-AKE molecules in their ground state $\left({ }^{2} \Sigma^{+}\right)$are shown. energies (Fig. 2). Also, the potential depths are different. In plot (b) of Fig. 3 the potential depths are shown and the values for molecules containing $\mathrm{Mg}$ are noticeably smaller than for the other AKE atoms, with no other trend being obvious for AKE atoms. However, there is a trend for AK atoms. The potential depth decreases seemingly exponentially in going from light to heavy AK atoms. The largest potential depth was determined for $\mathrm{LiCa}\left(2613 \mathrm{~cm}^{-1}\right)$, the smallest one for $\operatorname{RbMg}\left(744 \mathrm{~cm}^{-1}\right)$. In contrast to the PEDM and the equilibrium radii, the core polarization potential changes the potential depth in both directions. In cases where the AK atom is significantly lighter than the AKE atom the potential depth is reduced. However, if the AKE atom is lighter, the potential depth is increased. The equilibrium radii increase with inclusion of core polarization potentials. This effect is larger for heavier AK atoms, but shows no recognizable dependence on the AKE atom (ESI $\dagger$ ).

In contrast to the strictly positive PEDM for the doublet ground states the PEDMs for the lowest $\Sigma^{+}$state in the quartet manifold are negative, as can be seen in Fig. 4 . The PEDMs for the quartet states show a correlation with the difference in ENs (b) in Fig. 2. The most negative PEDM is found for the only molecule with a negative EN difference (LiSr), while small PEDMs are obtained for strongly positive EN differences. Small PEDMs of the ${ }^{4} \Sigma^{+}$state are observable in plot (a) in Fig. 4 for $\mathrm{Na}$ containing diatomic molecules including $\mathrm{NaBe}$ with the smallest value. This seems to be related to the first excitation energy of $\mathrm{Na}$, which is the largest of all AK atoms. The large excitation energy leads to the small - and for some combinations with $\mathrm{Na}$ even negative - differences in the excitation energy,

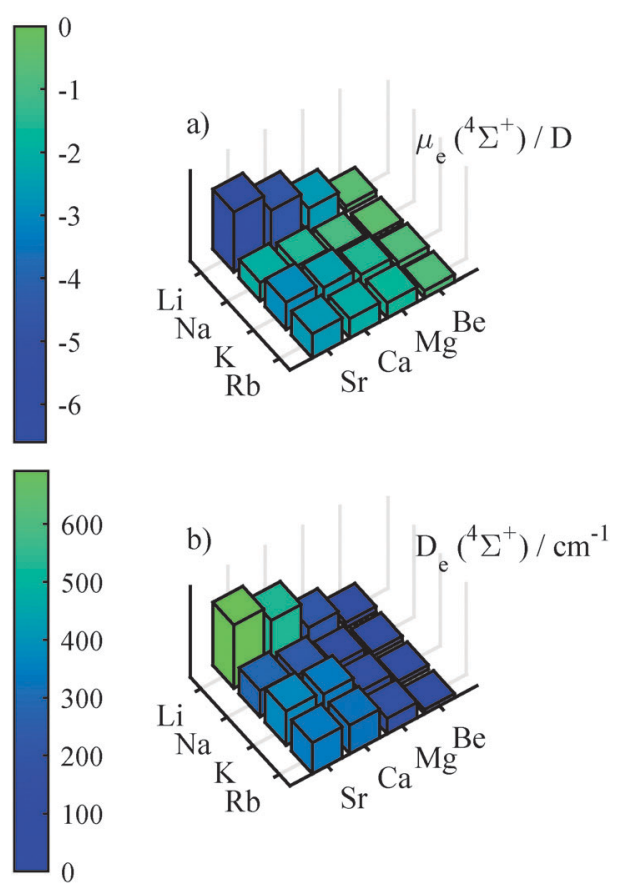

Fig. 4 The upper plot shows the PEDMs for several AK-AKE molecules in the lowest ${ }^{4} \Sigma^{+}$state. Note that the axis is reversed so that the highest bar corresponds to the most negative value. The graphics below shows the corresponding dissociation energy for a direct comparison. 
as can be seen in graphics (a) of Fig. 2. The potential depth turns out to be a reliable descriptor for the magnitude of the PEDM, as is evident by comparing plot (a) and (b) of Fig. 4, but is less useful for the estimation of PEDMs in the doublet manifold. For the ${ }^{4} \Sigma^{+}$state the dissociation energy and the PEDM have a correlation coefficient of $R=-0.97$.

The equilibrium internuclear distances of the lowest $\Sigma^{+}$ states in the two multiplicities are given in the Tables 2 and 3 . The $r_{\mathrm{e}}$ values for the doublet state are all below $4.57 \AA$, those for the quartet states lie above this value. The equilibrium distances can be compared to the tabulated covalent ${ }^{97}$ and van der Waals ${ }^{98}$ radii $(\mathrm{ESI} \dagger)$. The internuclear distances for the ground state minimum lie in between the covalent and van der Waals values. On average, they are off by $0.4 \AA$ from the covalent radii and by $1.4 \AA$ from the van der Waals radii. Them being closer to the covalent radii indicates a covalent or ionic bond in the lowest state of the doublet manifold. The lowest ${ }^{4} \Sigma^{+}$states have equilibrium internuclear separations that are on average $0.5 \AA$ larger than predicted by the tabulated van der Waals values, which indicates a weak bond.

The fraction of ionic character ${ }^{48}$ can be estimated by dividing the determined dipole moment by the dipole moment obtained by shifting one electron by the equilibrium distance: $f_{\text {Ion }}=\frac{\mu}{e \cdot r_{\mathrm{e}}}$. For the ground state, this fraction lies below 21\%, except for molecules containing Be, where the values are always above $21 \%$. As is obvious from the method of calculation, this quantity is correlated with the determined dipole moment and should be interpreted with caution. For the quartet states, particularly high values are observed for $\mathrm{Li}$ containing molecules.

With finite field calculations, which we performed in order to determine the dipole moment, we could also determine the polarizabilities of the molecules along the internuclear axis. Graphics (a) of Fig. 5 shows the polarizabilities for the ground state, which increase with atomic mass. These values are about 3.7 (with an variation from 3.4 to 4.0 ) times larger than the mean of the polarizability of the atoms involved. The polarizabilities for the quartet states show stronger variations (from 1.8 to 7.7 of the mean of the atomic pol.) than for the doublet states, as can be seen in graphics (b) of Fig. 5. Furthermore, there is a pronounced increase of polarizability for heavy AKE atoms, but no clear trend for the AK atoms. This dependence is probably related to the lowering of the first excitation energy when going from light to heavy AKE atoms. An increased mixing of the s-orbital of the AK atom and the p-orbital of the AKE atom facilitates a change in the electron density distribution.

\subsection{Qualitative picture}

Typical potential energy curves and PEDM curves for the lowest $\Sigma^{+}$states in the two multiplicities are shown in Fig. 6. The lowest ${ }^{2} \Sigma^{+}$is strongly bound with a positive PEDM. The PEDM approaches zero for large internuclear distances as expected for neutral molecules. In contrast, the lowest ${ }^{4} \Sigma^{+}$state is weakly bound with a negative PEDM. In general, the maximum $\left({ }^{2} \Sigma^{+}\right)$or minimum $\left({ }^{4} \Sigma^{+}\right)$of the PEDM curve is found for internuclear distances smaller than the equilibrium distance. The results

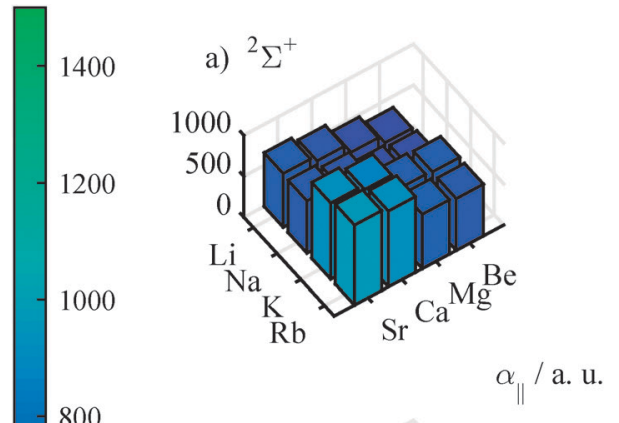

$-800$

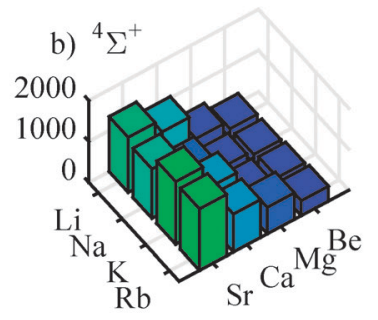

Fig. 5 Finite-field polarizabilities in the doublet ground state (upper graphics) and the lowest ${ }^{4} \Sigma^{+}$state (lower graphics), calculated the corresponding equilibrium geometry.
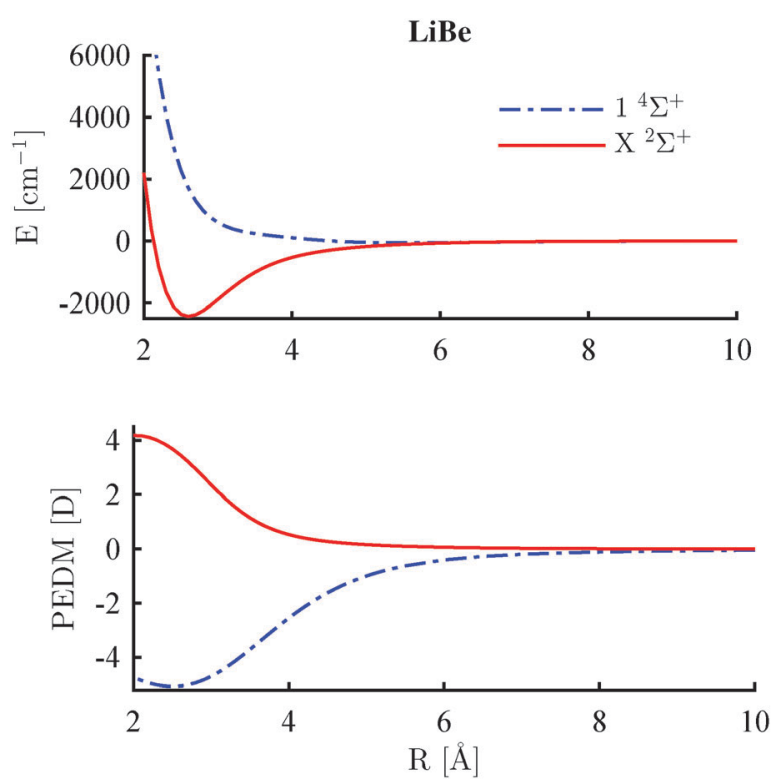

Fig. 6 Potentials and PEDMs of the lowest $\Sigma^{+}$state in the doublet and quartet multiplicity for the LiBe molecule.

displayed in Fig. 6 refer to LiBe, but the same behavior was previously observed for $\mathrm{LiCa}^{39}{ }^{3 \mathrm{RbCa}}{ }^{42}$ and $\mathrm{RbSr}^{43}$ In the current study, all 16 AK-AKE molecules confirm this trend. However, this behavior is not very pronounced for LiSr, and a different sign of the dipole moment can be expected in the ground state if $\mathrm{Ba}$ or Ra atoms are combined with Li. In fact, this has been observed by Gou et al. ${ }^{64}$

Our findings are summarized in Fig. 7. The ground state, displayed in graphics (a), shows a smaller bond distance. The dipole moment is positive, the vector of the dipole moment points from the AKE atom (++) to the AK atom (+). This is the 
a)
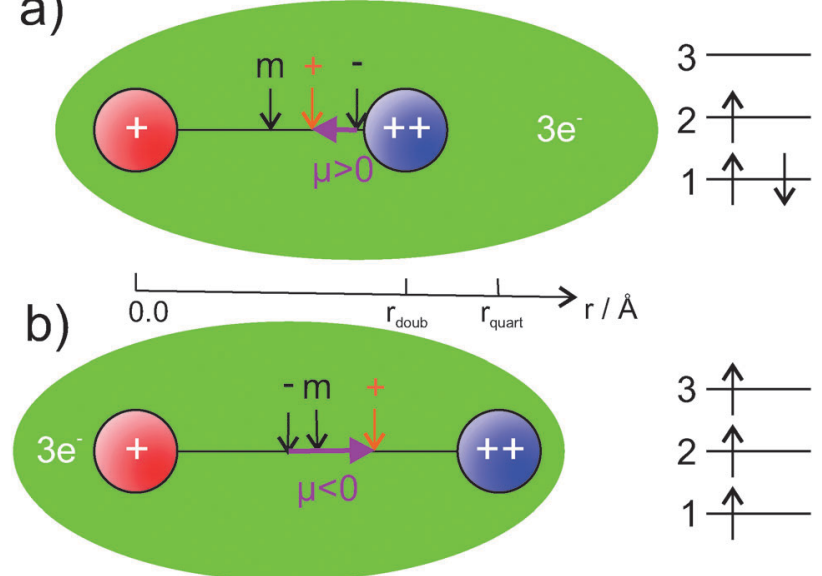

Fig. 7 A visualization of the charge distribution in our set of 16 AK-AKE molecules (no absolute scale). The AK (+) atom is shown in red, the AKE $(++)$ atom in blue. Center of mass, positive and negative charge are indicated by arrows. On the right side the orbital occupation discussed in the text is depicted. Calculated isosurfaces of the corresponding orbitals can be found in the ESI.

result of the center of charge of the electrons being closer to the AKE atom than the center of positive charge. The quartet state has a larger bond distance and a negative dipole moment. The center of electron charge is now closer to the AK atom than the center of positive charge. We explain this behavior by the orbital occupation indicated on the right side of Fig. 7. In the ground state (a) the 1st orbital, mainly an s-orbital around the AKE atom, $\|$ is doubly occupied. The 2nd orbital, mainly a mixture of s-orbitals from the AK atom and p-orbitals from the AKE atom, is singly occupied. Because the AKE orbital mixes into it, it is not centered at the AK atom. Therefore, the center of negative charge is closer to the AKE atom, and a positive PEDM is obtained in our coordinate system. This agrees with the situation indicated by the atomic polarizabilities. In general, the AK atoms have larger polarizabilities than AKE atoms. In the ground state, the highest occupied AK orbital should deform more easily and mix with AKE orbitals. The situation is different for the lowest $\Sigma^{+}$state in the quartet multiplicity (b) in Fig. 7. Since all spins need to be aligned, the Pauli principle does not allow for a double occupation of the 1st orbital. Therefore, one electron is transferred from this orbital into the 3rd orbital, which is a mixture of s-orbitals from the AK atom and p-orbitals from the AKE atom. For this reason, it has a probability density close to the AK atom, which changes the direction of the PEDM compared to the ground state. The 3rd orbital also mixes s- and p-orbital contributions in a similar way as the 2 nd orbital does. However, unlike the 2nd orbital, it has an additional node between the atoms, not only at the center of the AKE core.\| It corresponds, at least approximately, to the antibonding counterpart of the 2nd orbital, which explains the small dissociation energy of the lowest ${ }^{4} \Sigma^{+}$state.

\| The isosurfaces of the three orbitals indicated on the left side in Fig. 7 can be found in the ESI. $\dagger$

\subsection{Dipole moment predictions}

In this section we attempt to describe and predict the behavior of the dipole moment in the ground state due to its experimental importance for several applications mentioned in the introduction. The PEDMs of the lowest $\Sigma^{+}$state of the quartet multiplicity are well correlated with the dissociation energy, which is not the case for the ground state. However, we find the following empirical equation to reproduce the dipole moments surprisingly well for all species under investigation:

$$
\mu_{\mathrm{e}}=a_{1} \cdot \operatorname{sign}(\Delta \mathrm{EN}) \cdot \sqrt{|\Delta \mathrm{EN}|}+a_{2} \cdot \bar{P}_{\mathrm{at}}+\frac{a_{3}}{D_{\mathrm{e}}}
$$

This model requires the difference of electronegativities $\Delta \mathrm{EN}$, the mean of the atomic polarizabilities $\bar{P}_{\text {at }}$, and the dissociation energy $D_{\mathrm{e}}$ as input for the evaluation of the dipole moment. We note that it does not have yet a clear theoretical justification, as it was derived from an analysis of correlations between the dipole moment and atomic properties by a simple trial and error approach. However, it agrees very well with the available $a b$ initio data, as can be seen from Fig. 8, although it only requires three fitting parameters $a_{1}, a_{2}$ and $a_{3}$. At least the occurrence of the atomic descriptors in the formula can be readily justified. The difference in ENs accounts for the pull on the electron density, and the corresponding contribution to the dipole moment must therefore be related to a charge transfer. We do not have an explanation for the square root dependence, which made it necessary to also introduce the sign function in order to have the sign of the EN difference correlate with the direction of dipole moment. Because the value of the EN difference covers only a region from -0.1 to 0.9 other functions might also be suitable to capture the behavior. Slightly worse results are obtained for functions like $a_{3} \cdot\left(\Delta \mathrm{EN}-\frac{\Delta \mathrm{EN}^{2}}{2}\right)$ or the bounded $a_{3} \cdot \tanh (\Delta \mathrm{EN})$. The increase of the PEDM with increasing mean polarizability is easily understood by the greater spatial flexibility of the electron distribution. However, an explanation for the third term in eqn (1) is less straightforward. The corresponding fitting parameter $a_{3}$ takes a negative value in the model, therefore reducing the dipole moment proportional to the inverse of the corresponding dissociation energy. In other words, the weaker the bond, the more effectively this term reduces the dipole moment. This last contribution is not fully understood, but both properties are related to the spatial distribution of the orbitals.

This empirical model allows us to predict the dipole moments for other AK-AKE molecules. However, while the required ENs and the atomic polarizabilities can be found in ref. 95 and 96, the dissociation energies need to be estimated. For $\mathrm{Ba}$, the values from ref. 64 have been used. The potential depth of molecules including Cs or Fr were extrapolated with an exponential function from values for smaller AK atoms, since an exponential dependence was observed for the dissociation energy in Fig. 3. These results, together with previous theoretical results for $\mathrm{AK}-\mathrm{AKE}$ molecules beyond our test set, ${ }^{61,63,64}$ are shown in Fig. 8. We note that Gou et al. ${ }^{64}$ neglected the polarization of the cores, which might explain their larger amplitudes, 


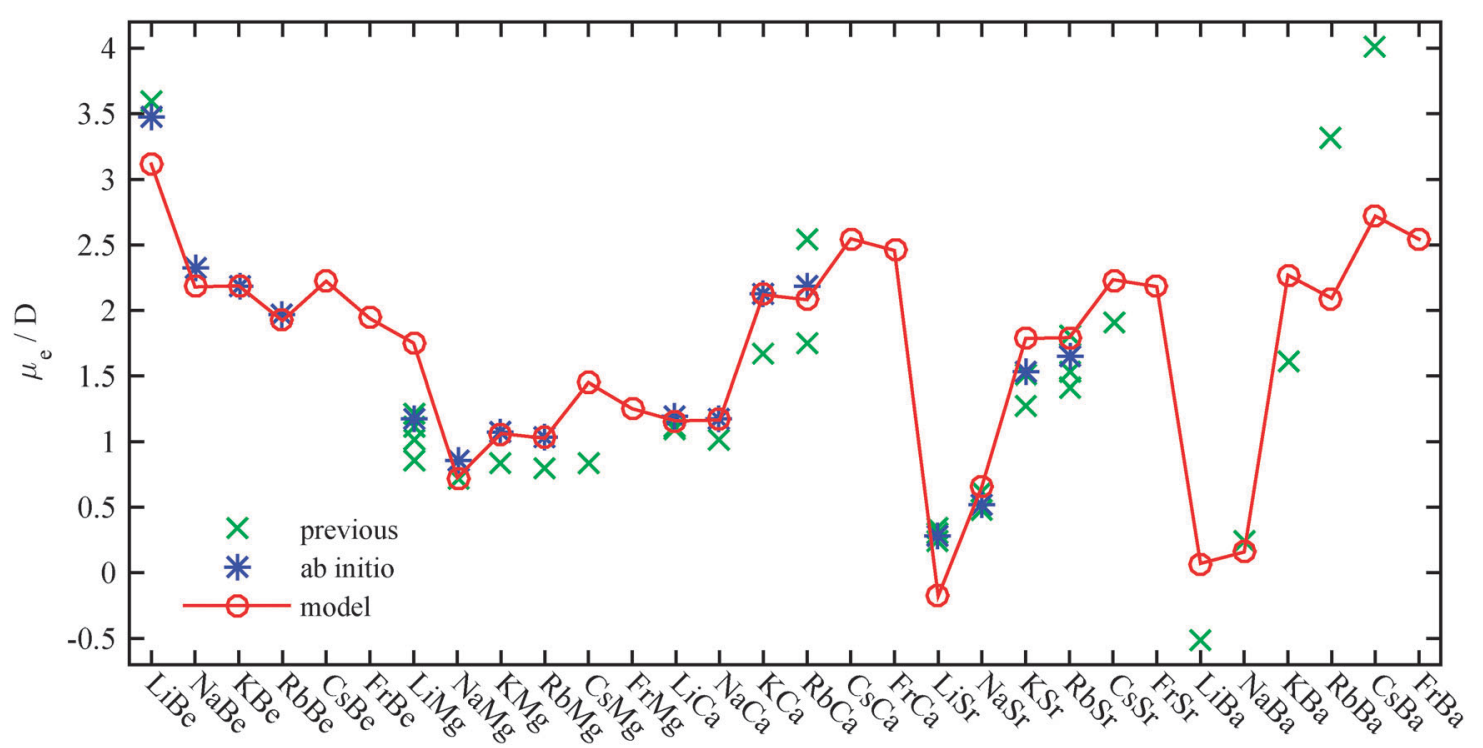

Fig. 8 Dipole moment predictions as described in the text $(O)$. The three parameters of the model were determined by fitting to the ab initio results of this work (*). The resulting model shows good agreement with results from previous theoretical treatments $(\times)$ listed in Table 2 . Additional values have been added for the CsMg (ref. 61), CsSr (ref. 63) and AK-Ba molecules (ref. 64).

similar to what we observed without core polarization potentials (Fig. 1). A detailed comparison between predicted and previous values is given in the ESI. $\dagger$

\section{Conclusion}

The lowest $\Sigma^{+}$states in the doublet and quartet manifold for a selection of 16 AK-AKE molecules were analyzed at the MCSCF + MRCI level of theory. Trends for the PEDMs, equilibrium internuclear separations, dissociation energies, and polarizabilities along the internuclear axis were discussed. We find that the PEDMs of the lowest $\Sigma^{+}$state in the doublet and quartet multiplicity are pointing in opposite directions for all molecules under investigation, which is in line with previous calculations. This behavior was explained in a molecular orbital picture. We further presented an empirical formula which describes the PEDM in the ${ }^{2} \Sigma^{+}$state as a function of two atomic descriptors (electronegativity, polarizability) and the dissociation energy. After fitting three parameters of the formula to our test set, we used this model to successfully predict the PEDM of several AK-AKE molecules beyond our initial test set.

The PEDM is important for applications of ultracold molecules, since it is the essential parameter for the interaction with microwaves, and, in combination with the polarizability, for the alignment in electric fields and optical lattices. A high PEDM is favorable because lower intensities and field strengths are sufficient to manipulate the molecules. In our study we find the highest PEDM for combinations with Be. However, handling these molecules might be a problem in the experiment due to the toxicity of beryllium. Good alternatives with high PEDM are $\mathrm{RbCa}, \mathrm{KCa}$, and $\mathrm{RbSr}$, with the latter being the most promising candidate at present. ${ }^{74}$ Our extrapolation formula indicates that CsCa might also be a promising choice.

\section{Acknowledgements}

The authors gratefully acknowledge support from NAWI Graz. This research was supported by the Austrian Science Fund (FWF) under Grant No. 22962-N20.

\section{References}

1 J. Ulmanis, J. Deiglmayr, M. Repp, R. Wester and M. Weidemuller, Chem. Rev., 2012, 112, 4890-4927.

2 G. Quéméner and P. S. Julienne, Chem. Rev., 2012, 112, 4949-5011.

3 I. Bloch and P. Zoller, in Ultracold Bosonic and Fermionic Gases, ed. A. L. F. Kathryn Levin and D. M. Stamper-Kurn, Elsevier, 2012, vol. 5, pp. 121-156.

4 L. D. Carr, D. DeMille, R. V. Krems and J. Ye, New J. Phys., 2009, 11, 055049.

5 J. Doyle, B. Friedrich, R. Krems and F. Masnou-Seeuws, Eur. Phys. J. D, 2004, 31, 149-164.

6 M. Baranov, L. Dobrek, K. Goral, L. Santos and M. Lewenstein, Phys. Scr., 2002, T102, 74-81.

7 L. Santos, G. V. Shlyapnikov, P. Zoller and M. Lewenstein, Phys. Rev. Lett., 2000, 85, 1791-1794.

8 K. Goral, L. Santos and M. Lewenstein, Phys. Rev. Lett., 2002, 88, 170406.

9 L. D. Carr, G. V. Shlyapnikov and Y. Castin, Phys. Rev. Lett., 2004, 92, 150404.

10 M. A. Baranov, L. Dobrek and M. Lewenstein, Phys. Rev. Lett., 2004, 92, 250403.

11 H. P. Buchler, E. Demler, M. Lukin, A. Micheli, N. Prokof'ev, G. Pupillo and P. Zoller, Phys. Rev. Lett., 2007, 98, 060404.

12 M. A. Baranov, M. Dalmonte, G. Pupillo and P. Zoller, Chem. Rev., 2012, 112, 5012-5061. 
13 D. DeMille, Phys. Rev. Lett., 2002, 88, 067901.

14 B. K. Stuhl, M. T. Hummon and J. Ye, Annu. Rev. Phys. Chem., 2014, 65, 501-518.

15 R. V. Krems, Phys. Chem. Chem. Phys., 2008, 10, 4079-4092.

16 E. R. Hudson, C. Ticknor, B. C. Sawyer, C. A. Taatjes, H. J. Lewandowski, J. R. Bochinski, J. L. Bohn and J. Ye, Phys. Rev. A: At., Mol., Opt. Phys., 2006, 73, 063404.

17 C. Chin, V. V. Flambaum and M. G. Kozlov, New J. Phys., 2009, 11, 055048.

18 M. Kajita, G. Gopakumar, M. Abe and M. Hada, J. Phys. B: At., Mol. Opt. Phys., 2013, 46, 025001.

19 M. Kajita, G. Gopakumar, M. Abe and M. Hada, J. Mol. Spectrosc., 2014, 300, 99-107.

20 V. V. Flambaum and M. G. Kozlov, Phys. Rev. Lett., 2007, 99, 150801.

21 E. R. Meyer and J. L. Bohn, Phys. Rev. A: At., Mol., Opt. Phys., 2009, 80, 042508.

22 E. R. Meyer, J. L. Bohn and M. P. Deskevich, Phys. Rev. A: At., Mol., Opt. Phys., 2006, 73, 062108.

23 K. Beloy, A. W. Hauser, A. Borschevsky, V. V. Flambaum and P. Schwerdtfeger, Phys. Rev. A: At., Mol., Opt. Phys., 2011, 84, 062114.

24 E. R. Hudson, H. J. Lewandowski, B. C. Sawyer and J. Ye, Phys. Rev. Lett., 2006, 96, 143004.

25 A. Fioretti, D. Comparat, A. Crubellier, O. Dulieu, F. Masnou-Seeuws and P. Pillet, Phys. Rev. Lett., 1998, 80, 4402-4405.

26 T. Takekoshi, B. M. Patterson and R. J. Knize, Phys. Rev. Lett., 1998, 81, 5105-5108.

27 T. Takekoshi, B. M. Patterson and R. J. Knize, Phys. Rev. A: At., Mol., Opt. Phys., 1999, 59, R5-R7.

28 A. N. Nikolov, E. E. Eyler, X. T. Wang, J. Li, H. Wang, W. C. Stwalley and P. L. Gould, Phys. Rev. Lett., 1999, 82, 703-706.

29 A. J. Kerman, J. M. Sage, S. Sainis, T. Bergeman and D. DeMille, Phys. Rev. Lett., 2004, 92, 153001.

30 A. J. Kerman, J. M. Sage, S. Sainis, T. Bergeman and D. DeMille, Phys. Rev. Lett., 2004, 92, 033004.

31 J. M. Sage, S. Sainis, T. Bergeman and D. DeMille, Phys. Rev. Lett., 2005, 94, 203001.

32 M. W. Mancini, G. D. Telles, A. R. L. Caires, V. S. Bagnato and L. G. Marcassa, Phys. Rev. Lett., 2004, 92, 133203.

33 D. Wang, J. Qi, M. F. Stone, O. Nikolayeva, H. Wang, B. Hattaway, S. D. Gensemer, P. L. Gould, E. E. Eyler and W. C. Stwalley, Phys. Rev. Lett., 2004, 93, 243005.

34 D. Wang, J. Qi, M. F. Stone, O. Nikolayeva, B. Hattaway, S. D. Gensemer, H. Wang, W. T. Zemke, P. L. Gould, E. E. Eyler and W. C. Stwalley, Eur. Phys. J. D, 2004, 31, 165-177.

35 A. J. Leggett, Rev. Mod. Phys., 2001, 73, 307-356.

36 S. Kraft, F. Vogt, O. Appel, F. Riehle and U. Sterr, Phys. Rev. Lett., 2009, 103, 130401.

37 S. Stellmer, M. K. Tey, B. Huang, R. Grimm and F. Schreck, Phys. Rev. Lett., 2009, 103, 200401.

38 Y. Takasu, K. Maki, K. Komori, T. Takano, K. Honda, M. Kumakura, T. Yabuzaki and Y. Takahashi, Phys. Rev. Lett., 2003, 91, 040404.
39 G. Krois, J. V. Pototschnig, F. Lackner and W. E. Ernst, J. Phys. Chem. A, 2013, 117, 13719-13731.

40 F. Lackner, G. Krois, T. Buchsteiner, J. V. Pototschnig and W. E. Ernst, Phys. Rev. Lett., 2014, 113, 153001.

41 G. Krois, F. Lackner, J. V. Pototschnig, T. Buchsteiner and W. Ernst, Phys. Chem. Chem. Phys., 2014, 16, 22373-22381.

42 J. V. Pototschnig, G. Krois, F. Lackner and W. E. Ernst, J. Chem. Phys., 2014, 141, 234309.

43 J. V. Pototschnig, G. Krois, F. Lackner and W. E. Ernst, J. Mol. Spectrosc., 2015, 310, 126-134.

44 J. Deiglmayr, M. Aymar, R. Wester, M. Weidemueller and O. Dulieu, J. Chem. Phys., 2008, 129, 064309.

45 G. Gopakumar, M. Abe, M. Hada and M. Kajita, J. Chem. Phys., 2014, 140, 224303.

46 A. Micheli, G. K. Brennen and P. Zoller, Nat. Phys., 2006, 2, 341-347.

47 S. Kotochigova, Phys. Rev. Lett., 2007, 99, 073003.

48 E. S. Rittner, J. Chem. Phys., 1951, 19, 1030-1035.

49 P. Brumer and M. Karplus, J. Chem. Phys., 1973, 58, 3903-3918.

50 R. L. Matcha and S. C. King, J. Am. Chem. Soc., 1976, 98, 3420-3432.

51 T. Törring, W. E. Ernst and J. Kändler, J. Chem. Phys., 1989, 90, 4927-4932.

52 T. Törring, W. E. Ernst and S. Kindt, J. Chem. Phys., 1984, 81, 4614-4619.

53 S. F. Rice, H. Martin and R. W. Field, J. Chem. Phys., 1985, 82, 5023-5034.

54 J. Schamps, M. Bencheikh, J. C. Barthelat and R. W. Field, J. Chem. Phys., 1995, 103, 8004-8013.

55 H. Schall, M. Dulick and R. W. Field, J. Chem. Phys., 1987, 87, 2898-2912.

56 W. E. Ernst and J. O. Schröder, Z. Phys. D: At., Mol. Clusters, 1986, 1, 103-112.

57 S. Hou and P. F. Bernath, J. Phys. Chem. A, 2015, 119, 1435-1438.

58 C. W. Bauschlicher, S. R. Langhoff and H. Partridge, J. Chem. Phys., 1992, 96, 1240-1247.

59 G. Gopakumar, M. Abe, M. Kajita and M. Hada, Phys. Rev. A: At., Mol., Opt. Phys., 2011, 84, 062514.

60 S. Kotochigova, A. Petrov, M. Linnik, J. Klos and P. S. Julienne, J. Chem. Phys., 2011, 135, 164108.

61 R. Guerout, M. Aymar and O. Dulieu, Phys. Rev. A: At., Mol., Opt. Phys., 2010, 82, 042508.

62 M. Aymar, R. Guerout and O. Dulieu, J. Chem. Phys., 2011, 135, 064305.

63 L. Augustovičová and P. Soldán, J. Chem. Phys., 2012, 136, 084311.

64 D. Z. Gou, X. Y. Kuang, Y. F. Gao and D. M. Huo, J. Chem. Phys., 2015, 142, 034308.

65 A. R. Allouche and M. Aubert-Frécon, J. Chem. Phys., 1994, 100, 938-944.

66 J. D’Incan, C. Effantin, A. Bernard, G. Fabre, R. Stringat, A. Boulezhar and J. Vérges, J. Chem. Phys., 1994, 100, 945-949.

67 N. Boutassetta, A. R. Allouche and M. Aubert-Frécon, Chem. Phys., 1994, 189, 33-39. 
68 M. Ivanova, A. Stein, A. Pashov, A. V. Stolyarov, H. Knockel and E. Tiemann, J. Chem. Phys., 2011, 135, 174303.

69 A. Stein, M. Ivanova, A. Pashov, H. Knöckel and E. Tiemann, J. Chem. Phys., 2013, 138, 114306.

70 C. H. Wu, H. R. Ihle and K. A. Gingerich, Int. J. Mass Spectrom., 1983, 47, 235-238.

71 A. R. Allouche and M. Aubert-Frécon, Chem. Phys. Lett., 1994, 222, 524-528.

72 G. Gopakumar, M. Abe, M. Hada and M. Kajita, J. Chem. Phys., 2013, 138, 194307.

73 L. M. Russon, G. K. Rothschopf, M. D. Morse, A. I. Boldyrev and J. Simons, J. Chem. Phys., 1998, 109, 6655-6665.

74 B. Pasquiou, A. Bayerle, S. M. Tzanova, S. Stellmer, J. Szczepkowski, M. Parigger, R. Grimm and F. Schreck, Phys. Rev. A: At., Mol., Opt. Phys., 2013, 88, 023601.

75 P. S. Zuchowski, J. Aldegunde and J. M. Hutson, Phys. Rev. Lett., 2010, 105, 153201.

76 T. Chen, S. B. Zhu, X. L. Li, J. Qian and Y. Z. Wang, Phys. Rev. A: At., Mol., Opt. Phys., 2014, 89, 063402.

77 P. S. Zuchowski, R. Guerout and O. Dulieu, Phys. Rev. A: At., Mol., Opt. Phys., 2014, 90, 012507.

78 S. Knecht, L. K. Sorensen, H. J. A. Jensen, T. Fleig and C. M. Marian, J. Phys. B: At., Mol. Opt. Phys., 2010, 43, 055101.

79 Y. F. Gao and T. Gao, Mol. Phys., 2014, 112, 3015-3023.

80 H.-J. Werner, P. J. Knowles, G. Knizia, F. R. Manby, M. Schütz, P. Celani, T. Korona, R. Lindh, A. Mitrushenkov, G. Rauhut, K. R. Shamasundar, T. B. Adler, R. D. Amos, A. Bernhardsson, A. Berning, D. L. Cooper, M. J. O. Deegan, A. J. Dobbyn, F. Eckert, E. Goll, C. Hampel, A. Hesselmann, G. Hetzer, T. Hrenar, G. Jansen, C. Köppl, Y. Liu, A. W. Lloyd, R. A. Mata, A. J. May, S. J. McNicholas, W. Meyer, M. E. Mura, A. Nicklass, D. P. O’Neill, P. Palmieri, D. Peng, K. Pflüger, R. Pitzer, M. Reiher, T. Shiozaki, H. Stoll, A. J. Stone, R. Tarroni, T. Thorsteinsson and M. Wang, MOLPRO, version 2012.1, a package of ab initio programs, 2012, see http://www.molpro.net.
81 P. J. Knowles and H.-J. Werner, Chem. Phys. Lett., 1985, 115, 259-267.

82 H.-J. Werner and P. J. Knowles, J. Chem. Phys., 1985, 82, 5053.

83 H.-J. Werner and P. J. Knowles, J. Chem. Phys., 1988, 89, 5803-5814.

84 P. J. Knowles and H.-J. Werner, Theor. Chem. Acc., 1992, 84, 95-103.

85 B. P. Prascher, D. E. Woon, K. A. Peterson, T. H. Dunning and A. K. Wilson, Theor. Chem. Acc., 2011, 128, 69-82.

86 H. D. Li, H. Feng, W. G. Sun, Y. Zhang, Q. C. Fan, K. A. Peterson, Y. M. Xie and H. F. Schaefer, Mol. Phys., 2013, 111, 2292-2298.

87 I. S. Lim, P. Schwerdtfeger, B. Metz and H. Stoll, J. Chem. Phys., 2005, 122, 104103.

88 I. S. Lim, H. Stoll and P. Schwerdtfeger, J. Chem. Phys., 2006, 124, 034107.

89 W. Müller, J. Flesh and W. Meyer, J. Chem. Phys., 1984, 80, 3297-3310.

90 W. Müller and W. Meyer, J. Chem. Phys., 1984, 80, 3311-3320.

91 D. A. Fedorov, A. Derevianko and S. A. Varganov, J. Chem. Phys., 2014, 140, 184315.

92 Y. Ralchenko, A. Kramida, J. Reader and NIST ASD Team, National Institute of Standards and Technology, Gaithersburg, MD, 2015, available: http://physics.nist.gov/asd.

93 R. Schlachta, I. Fischer, P. Rosmus and V. E. Bondybey, Chem. Phys. Lett., 1990, 170, 485-491.

94 K. R. Berry and M. A. Duncan, Chem. Phys. Lett., 1997, 279, 44-49.

95 J. Mitroy, M. S. Safronova and C. W. Clark, J. Phys. B: At., Mol. Opt. Phys., 2010, 43, 202001.

96 CRC Handbook of Chemistry and Physics, ed. D. R. Lide, CRC Press, 85th edn, 2004.

97 B. Cordero, V. Gomez, A. E. Platero-Prats, M. Reves, J. Echeverria, E. Cremades, F. Barragan and S. Alvarez, Dalton Trans., 2008, 2832-2838.

98 S. Alvarez, Dalton Trans., 2013, 42, 8617-8636. 